\title{
Colchicine for cardiovascular therapy: A drug interaction perspective and a safety meta-analysis
}

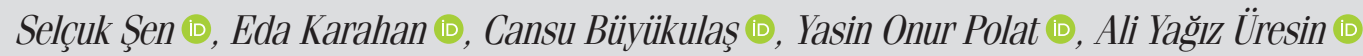 \\ Division of Clinical Pharmacology, Department of Medical Pharmacology, İstanbul Faculty of Medicine, \\ İstanbul University; İstanbul-Turkey
}

ABSTRACT

Objective: In this study, we aimed to overview the drug interactions between colchicine and cardiovascular drugs and performed a metaanalysis to evaluate the safety profile of colchicine in cardiovascular treatment.

Methods: The drug interactions between colchicine and cardiovascular drugs were evaluated using Medscape-Drug-Interaction-checker. For safety meta-analysis, a systematic literature search was carried out to retrieve eligible studies. Randomized controlled clinical trials that reported a safety analysis and with at least one-year follow-up period were included into the analysis. Meta-analysis was performed using RevMan 5.4 software provided by the Cochrane Collaboration.

Results: Serious drug interactions were found between colchicine and lipid-lowering treatments, including all statins and fibrates; carvedilol among the beta-blockers; non-dihydropyridine calcium channel blockers (verapamil and diltiazem); and amiodarone, digoxin, and quinidine. The safety meta-analysis involved 11,594 patients with coronary disease from four trials. The incidences of gastrointestinal adverse events; hematological adverse events; infection; pneumonia; cancer; myalgia; and abnormal nerve sensations were similar between colchicine and control arm. The incidence of cardiovascular death was lower in the colchicine arm; however, the difference did not reach a significant level [relative risk (RR): $0.71,95 \%$ confidence interval (CI) $0.48-1.05 ; \mathrm{p}=0.09$ ]. The incidence of non-cardiovascular death was significantly higher in the colchicine arm (RR: $1.53,95 \% \mathrm{Cl} 1.10-2.14 ; \mathrm{p}=0.01$ ). The rate of all-cause mortality was similar between the two arms (RR: $1.04,95 \% \mathrm{Cl} 0.61-1.78 ; \mathrm{p}=0.88$ ).

Conclusion: Cardiac patients on colchicine therapy should be carefully monitored to avoid the complications of serious drugs interactions. The reported data in the literature were not sufficient to evaluate the common side effects such as gastrointestinal events and myalgia. The higher incidence of non-cardiovascular death cannot be ignored and should be thoroughly investigated in future studies.

Keywords: colchicine, cardiovascular, coronary disease, drug interaction, safety, non-cardiovascular death

Cite this article as: Şen S, Karahan E, Büyükulaş C, Polat YO, Üresin AY. Colchicine for cardiovascular therapy: A drug interaction perspective and a safety meta-analysis. Anatol J Cardiol 2021; 25: 753-61.

\section{Introduction}

There is a strong association between inflammation and the development of atherosclerosis (1). In addition to the effects of lipid lowering treatments, investigating the potential effects of anti-inflammatory drugs on atherosclerosis and related cardiovascular diseases has become an important translational research field. However, all anti-inflammatory drugs do not have beneficial effects on the cardiovascular system; some can cause adverse effects as observed with cyclooxygenase-2 (COX-2) selective non-steroidal anti-inflammatory drugs in the past. In addition, in the Cardiovascular Inflammation Reduction Trial (CIRT), the effect of an alternative drug, low-dose methotrexate, on cardiovascular outcomes was evaluated; however, the low-dose methotrexate did not reduce the rate of cardiovascular events, and no significant reduction was reported in inflammation related biomarkers including interleukin (IL) $1 \beta$, IL-6, and C-reactive protein (2). In

Address for Correspondence: Dr. Selçuk Şen, İstanbul Üniversitesi İstanbul Tıp Fakültesi,

Tıbbi Farmakoloji Anabilim Dalı, İstanbul-Türkiye

Phone: +90 2124142240 E-mail: slcsen@istanbul.edu.tr

Accepted Date: 31.08.2021 Available Online Date: 08.10.2021

(C) Copyright 2021 by Turkish Society of Cardiology - Available online at www.anatoljcardiol.com DOI:10.5152/AnatolJCardiol.2021.707 


\section{HIGHLIGHTS}

- Colchicine has serious risks of drug interaction with some cardiovascular drugs, including statins.

- Low-dose colchicine $(0.5 \mathrm{mg} /$ day) in cardiovascular treatment can generally be considered safe; however, the existing data are not sufficient to reach a definitive conclusion.

- The higher incidence of non-cardiovascular death (relative risk: $1.53,95 \%$ confidence interval 1.10-2.14; $\mathrm{p}=0.01$ ) cannot be ignored and should be thoroughly investigated.

recent years, the relationship between the IL-1 $\beta$ inhibition, inflammation, and cardiovascular outcomes has become a leading research topic. The Canakinumab Anti-Inflammatory Thrombosis Outcomes Study (CANTOS) has shown that canakinumab, a monoclonal antibody targeting the IL-1 $\beta$, significantly reduced recurrent cardiovascular events with an increased risk of fatal infection than the placebo (3). Among commonly used anti-inflammatory drugs, colchicine, has emerged as an inexpensive potential treatment option, which may contribute to additional benefits.

In addition to its use in the treatment of gout, colchicine can be used in the treatment of several other conditions including familial Mediterranean fever (FMF) because of its multiple antiinflammatory properties (4). The main mechanism of action of colchicine is inhibition of microtubule polymerization by binding to the tubulin molecule (5). However, it is now suggested that colchicine may have multiple properties indicating a more complex anti-inflammatory mechanism of action than previously defined (4). The effects of colchicine through inhibiting the NLRP3 inflammasome function (6); suppression of the proinflammatory M1 macrophages and upregulation of anti-inflammatory M2 macrophages (7); and inhibition of leukocyte-platelet aggregation (8) are believed to be the possible mechanisms underlying cardiovascular benefits.

The Colchicine Cardiovascular Outcomes Trial (COLCOT) shed new light on the topic of colchicine use in cardiovascular treatment, revealing that low-dose colchicine $0.5 \mathrm{mg} /$ day significantly reduces the risk of ischemic cardiovascular events in the treatment of patients with a prior myocardial infarction (9). Moreover, initiation of colchicine treatment earlier in the hospital may provide better outcomes (10). The findings in the COLCOT trial was also supported by the results of the more recent Low Dose Colchicine for Secondary Prevention of Cardiovascular disease 2 (LoDoCo2) study (11).

Management of drug interactions is one of the most important challenges in the treatment of chronic and complex health conditions. The inappropriate use of colchicine combined with the presence of polypharmacy in cardiac patients can result in significant risk of drug interactions. Colchicine can interact with other drugs, especially through p-glycoprotein and cytochrome
3A4 (CYP3A4) pathways. The physicians will probably be confronted with the use of colchicine in the treatment of cardiovascular diseases. In this study, we aimed to overview the drug interactions between colchicine and the drugs that are commonly used in cardiovascular treatment. In addition, a metaanalysis of randomized clinical trials was performed to evaluate the safety profile of colchicine in the treatment of coronary disease and the risk of adverse events.

\section{Methods}

The drug interactions between colchicine and cardiovascular drugs were overviewed using online Medscape Drug Interaction checker (12). Eighty-three cardiovascular drugs of interest including anticoagulants, antiplatelet drugs, angiotensin converting enzyme (ACE) inhibitors, angiotensin II receptor blockers (ARBs), angiotensin receptor/neprilysin inhibitor, betablockers, calcium channel blockers, statins, other cholesterol lowering drugs, digitalis preparations, diuretics, and vasodilator agents, and other cardiovascular drugs are listed in supplementary material (Table S1). According to Medscape drug interaction checker (12), the drug interaction was classified into contraindicated, serious (use an alternative drug), significant (monitor closely), and minor.

For safety meta-analysis, the search was carried out by 2 researchers using the keywords "colchicine" and ("coronary artery disease" or "coronary heart disease" or "ischemic heart disease" or "myocardial infarction" or "angina" or "acute coronary syndrome") in PubMed and Cochrane Central Register of Controlled Clinical Trials databases till July 2021. Moreover, the references from the selected articles and other systematic reviews were checked to retrieve any initially missed studies. Randomized controlled clinical trials that have reported a safety analysis and with an at least one-year follow-up period were included in the analysis. Meta-analysis was conducted using RevMan 5.4 provided by the Cochrane Collaboration. MantelHaenszel weighting method was used, and dichotomous data were expressed as relative risk (RR) with $95 \%$ confidence interval. Heterogeneity was assessed by visual assessment of forest plots, chi-squared $p$ value, and the value of Higgins Index $\left(1^{2}\right)$ according to Cochrane Handbook Criteria (13). A random-effects model was used if heterogeneity was considered as high $(12 \geq 50 \%$ and/or chi-squared $p$ value $\leq 0.10)$, whereas a fixedeffects model was used if heterogeneity was considered as low $\left(\mathrm{I}^{2}<50 \%\right.$ and chi-squared $\mathrm{p}$ value $\left.>0.10\right)$. The Cochrane Risk of Bias tool was used to evaluate methodological quality of each study with the aspects, including random sequence generation (selection bias), allocation concealment (selection bias), blinding of participants and personnel (performance bias), blinding of outcome assessment (detection bias), incomplete outcome data (attrition bias), and selective reporting (reporting bias). Evaluation of publication bias was not performed owing to the low number of included studies. The results were reported in accordance with Preferred Reporting Items for Systematic reviews and Meta-analysis guidelines (14). 


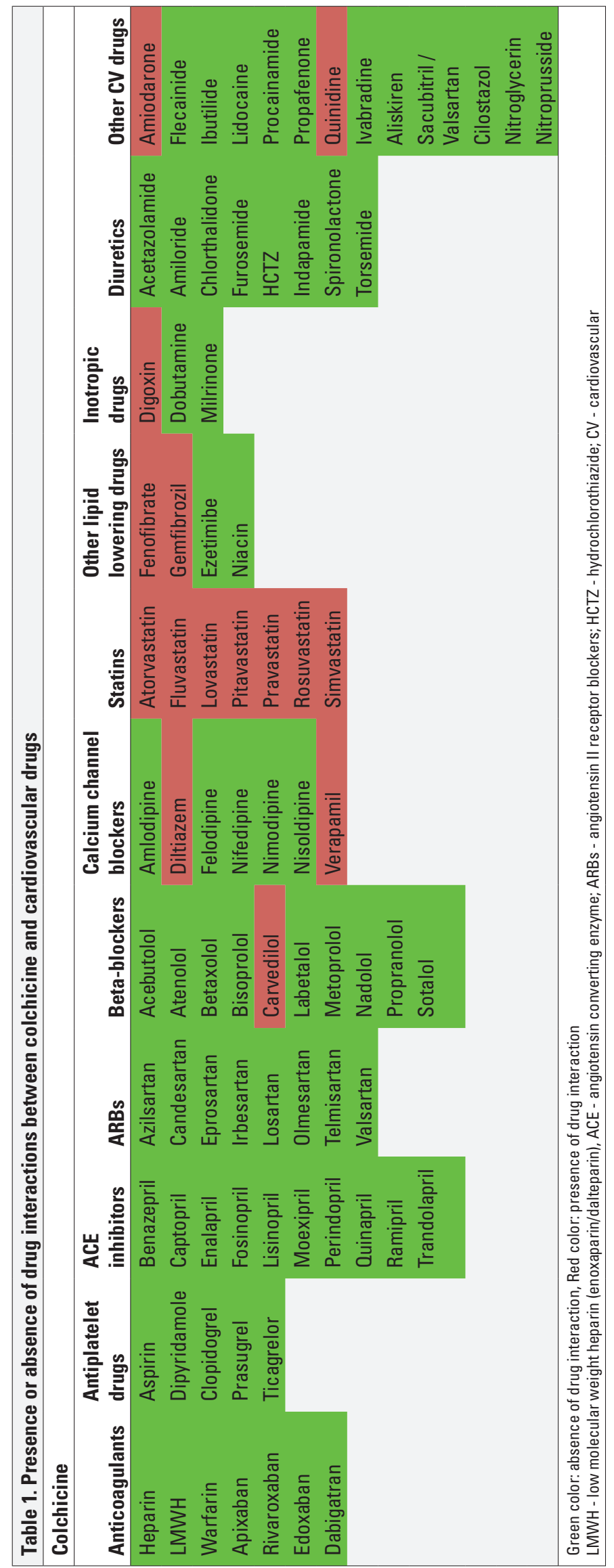

\section{Results}

\section{Overview of drug interactions between colchicine and cardiovascular drugs}

First, the presence or absence of drug interactions between colchicine and cardiovascular drugs were extracted using Medscape Drug Interaction Checker (12). It has been determined that colchicine interacts with 15 cardiovascular drugs among the total of 83 evaluated drugs (Table 1). Drug interactions were found between colchicine and lipid lowering treatments, including all statins and fibrates; carvedilol among the beta-blockers; non-dihidropyridine calcium channel blockers including verapamil and diltiazem; and amiodarone, digoxin, and quinidine. Identified drug interactions were analyzed in detail in terms of the interaction type (pharmacokinetic/pharmacodynamic), mechanism, severity, and recommendation. The findings are given in Table 2. All of the identified drug interactions were serious, and it was generally recommended to use alternative drugs. In terms of the drug interaction type and mechanism, statins did not differ from each other.

\section{Search results of safety meta-analysis}

Through literature search, 432 articles were retrieved. Of these, 28 duplicate records were removed. Of the 404 remaining records, 391 were excluded after screening owing to irrelevant title and/or abstracts. The remaining 13 articles were assessed for eligibility and inclusion criteria. Of these, 9 articles were excluded for not meeting the inclusion criteria. Four randomized controlled clinical trials were included in the final analysis $(9,11$, $15,16)$. The literature search and exclusion of articles at each step are summarized in the supplementary material (Fig. S1).

\section{Characteristics of included studies in meta-analysis}

Table 3 summarizes the baseline characteristics of the included clinical trials published between 2013 and 2020. The analysis involved 11,594 patients with coronary disease, with 5,806 of them having received colchicine treatment and 5,788 of them assigned to the active control arm. All trials were randomized controlled clinical trials. The intervention duration of the trials ranged from 12 to 36 months. The rate of statin use was evaluated for each study and ranged from $94 \%$ to $99 \%$. In the LoDoCo (16) trial published in 2013, the incidences of adverse events such as gastrointestinal adverse events, muscle related toxicity, and so forth, were reported only for the colchicine arm but not for the control arm; therefore, from the LoDoCo study, only the mortality outcomes were used for the meta-analysis, being available for both arms.

\section{Risk of bias assessment}

The risk of bias assessment was conducted for all studies separately. All the studies were considered to carry a low risk of random sequence generation (selection bias), allocation concealment (selection bias), blinding of outcome assessment (detection bias), incomplete outcome data (attrition bias), and selective reporting (reporting bias). All the studies were of dou- 


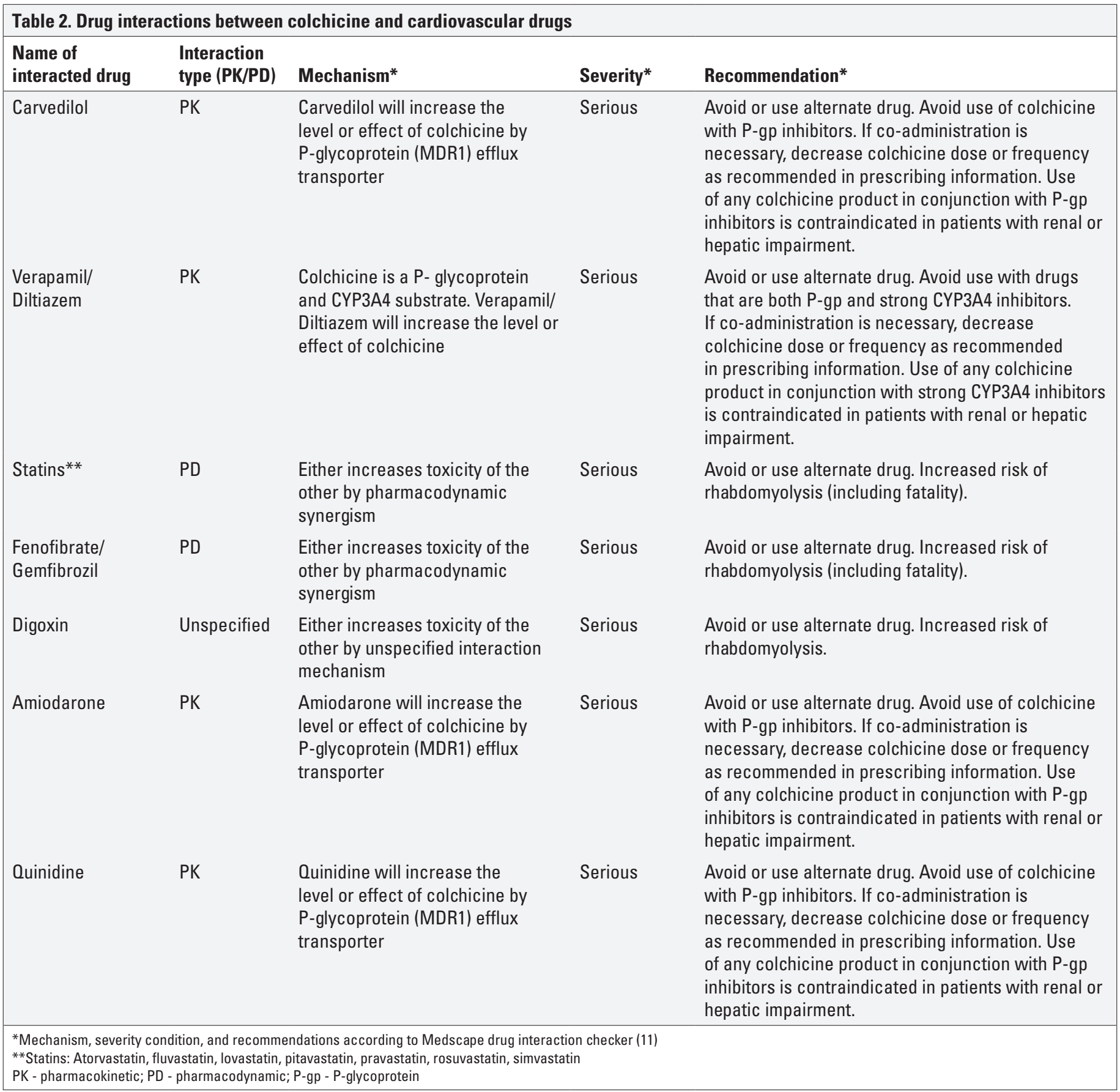

ble-blind design, except for the LoDoCo study published in 2013 (16). Differently from other included studies, placebo was not used in the control arm of the LoDoCo study, which may cause a high risk of bias in the blinding of participants and personnel (performance bias). There was a baseline balance for the treatment and the control arms in all studies. The risk of bias assessment is summarized in supplementary material (Fig. S2).

\section{Findings in the safety meta-analysis}

High heterogeneity $\left(\mid I^{2} \geq 50 \%\right.$ and/or chi-squared $p$ value $\leq 0.10)$ across the studies were identified in the reported inci- dence of infection, pneumonia, myalgia, and all-cause mortality; whereas low heterogeneity $\left(I^{2}<50 \%\right.$ and chi-squared $p$ value $>0.10$ ) was identified in the reported incidence of gastrointestinal adverse events, hematological adverse events, cancer, abnormal nerve sensations, hospitalization for heart failure, gout, cardiovascular death, and non-cardiovascular death. Incidences of gastrointestinal adverse events (RR: $1.02,95 \% \mathrm{Cl}$ 0.91-1.13; $\mathrm{p}=0.78$ ); hematological adverse events (RR: 1.01, 95\% Cl 0.57-1.79; $\mathrm{p}=0.98$ ); infection (RR: $1.08,95 \% \mathrm{Cl} 0.78-1.51$; $\mathrm{p}=0.64$ ); pneumonia (RR: $1.47,95 \% \mathrm{Cl} 0.57-3.79 ; \mathrm{p}=0.42$ ); cancer (RR: $0.98,95 \% \mathrm{Cl} 0.80-1.21 ; \mathrm{p}=0.88$ ); myalgia (RR: $0.37,95 \% \mathrm{Cl}$ 


\begin{tabular}{|c|c|c|c|c|c|c|c|c|c|c|}
\hline $\begin{array}{l}\text { Study } \\
\text { name }\end{array}$ & Year & $\begin{array}{l}\text { Number of } \\
\text { participants } \\
\text { included } \\
\text { in safety } \\
\text { analysis }\end{array}$ & \multicolumn{2}{|c|}{ Age } & \multicolumn{2}{|c|}{ Female sex } & $\begin{array}{l}\text { Medical } \\
\text { condition }\end{array}$ & $\begin{array}{l}\text { Colchicine } \\
\text { dose }\end{array}$ & $\begin{array}{l}\text { Statin } \\
\text { use } \\
(\%)\end{array}$ & $\begin{array}{l}\text { Follow-up } \\
\text { (months) }\end{array}$ \\
\hline $\begin{array}{l}\text { COLCOT } \\
\text { (9) }\end{array}$ & 2019 & 4745 & $60.6 \pm 10.7$ & $60.5 \pm 10.6$ & $472(19.9 \%)$ & $437(18.4 \%)$ & $\begin{array}{l}\text { Post myocardial } \\
\text { infarction within } \\
30 \text { days }\end{array}$ & $0.5 \mathrm{mg} / \mathrm{day}$ & 99 & 20 \\
\hline $\begin{array}{l}\text { COPS } \\
(15)\end{array}$ & 2020 & 795 & $59.7 \pm 10.2$ & $60.0 \pm 10.4$ & $74(18.6 \%)$ & $89(22.3 \%)$ & $\begin{array}{l}\text { Acute coronary } \\
\text { syndrome }\end{array}$ & $\begin{array}{l}0.5 \mathrm{mg} \text { twice } \\
\text { daily for } \\
\text { a month, } \\
\text { followed by } \\
0.5 \mathrm{mg} / \mathrm{day}\end{array}$ & 99 & 12 \\
\hline $\begin{array}{l}\text { LoDoCo2 } \\
\text { (11) }\end{array}$ & 2020 & 5522 & $65.8 \pm 8.4$ & $65.9 \pm 8.7$ & $457(16.5 \%)$ & $389(14.1 \%)$ & $\begin{array}{l}\text { Chronic } \\
\text { coronary } \\
\text { disease }\end{array}$ & $0.5 \mathrm{mg} / \mathrm{day}$ & 94 & 28.6 \\
\hline
\end{tabular}

0.02-6.36; $\mathrm{p}=0.49$ ); abnormal nerve sensations (RR: $0.94,95 \% \mathrm{Cl}$ $0.75-1.17 ; p=0.57$ ) were not significantly different between the colchicine and the control arms. The incidence of hospitalization for heart failure was only reported in COLCOT study, and there was no difference between the colchicine and the control arms (RR: $1.48,95 \% \mathrm{Cl} 0.80-2.73 ; \mathrm{p}=0.21$ ). The incidence of gout was only reported in LoDoCo2 trial, and the incidence was significantly lower in colchicine arm than in the control (RR: 0.40 , 95\% Cl 0.28-0.58; $p<0.00001$ ) (Fig. 1).

Mortality outcomes are presented in Figure 2. Although it did not reach a significant level, the incidence of cardiovascular death was lower in the colchicine arm than in the control (RR: $0.71,95 \% \mathrm{Cl} 0.48-1.05 ; \mathrm{p}=0.09$ ). However, the incidence of noncardiovascular death was significantly higher in the colchicine arm than in the control (RR: $1.53,95 \% \mathrm{Cl} 1.10-2.14 ; \mathrm{p}=0.01$ ). Finally, there was no difference between the colchicine and control arms in terms of all-cause mortality (RR: $1.04,95 \% \mathrm{Cl}$ $0.61-1.78 ; p=0.88$ ).

\section{Discussion}

In the COLCOT trial published in 2019, a total of 4,745 patients who had a myocardial infarction within the last 30 days were randomized to low-dose $(0.5 \mathrm{mg} /$ day) colchicine and placebo arms and followed up for a median of 22.6 months. The composite primary endpoint of the COLCOT trial was to compare the rate of cardiovascular death, resuscitated cardiac arrest, myocardial infarction, stroke, or urgent hospitalization for angina leading to coronary revascularizations between the study arms. The incidence of primary endpoint was reported to be significantly lower in the colchicine arm than in the placebo $(5.5 \%$ vs. $7.1 \%$, respectively; hazard ratio $0.77 ; 95 \% \mathrm{Cl} 0.61-0.96 ; \mathrm{p}=0.02$ ) (9). In a later evaluation of the COLCOT study, it was also reported that the time-to-treatment initiation influenced the treatment suc- cess, and low-dose colchicine treatment may be more beneficial if initiated early in hospital (10). More recently, the results of the LoDoCo2 study have been published, and this study reached a conclusion that low-dose colchicine $0.5 \mathrm{mg} /$ day can reduce the risk of cardiovascular events in patients with chronic coronary disease (11). With the results of large trials, the off-label use of colchicine or the patients who are already using colchicine for other approved conditions will probably be the matter of the cardiovascular area. In this study, the drug interactions between colchicine and most commonly prescribed cardiovascular drugs were evaluated to provide physicians with guidance with regard to warnings and precautions. A direct contraindication between colchicine and any of the cardiovascular drugs was not listed. However, the serious interactions should be taken into consideration before the co-administration of colchicine with cardiovascular drugs. The most commonly used lipid lowering treatments including all statins and fibrates but not with ezetimibe and niacin; carvedilol among beta-blockers; nondihidropyridine calcium channel blockers including verapamil and diltiazem; and amiodarone, digoxin, and quinidine have serious drug interactions with colchicine. In some patients, the concomitant drug may be stopped and/or replaced by an alternative treatment, such as carvedilol can be replaced by an appropriate beta-blocker or non-dihidroprydines can be replaced with other drugs if available, or colchicine treatment can be stopped if the concomitant treatment will be used for a short term. However, if the concomitant treatment is essential and carries out an overwhelming benefit for the patients, such as statins, it can be necessary to continue the treatment. These patients should be monitored closely with both clinical and laboratory findings.

The recommendation of Medscape drug interaction database is to avoid the co-administration of colchicine with all statins or to use an alternative treatment. It is very well-known that statins 


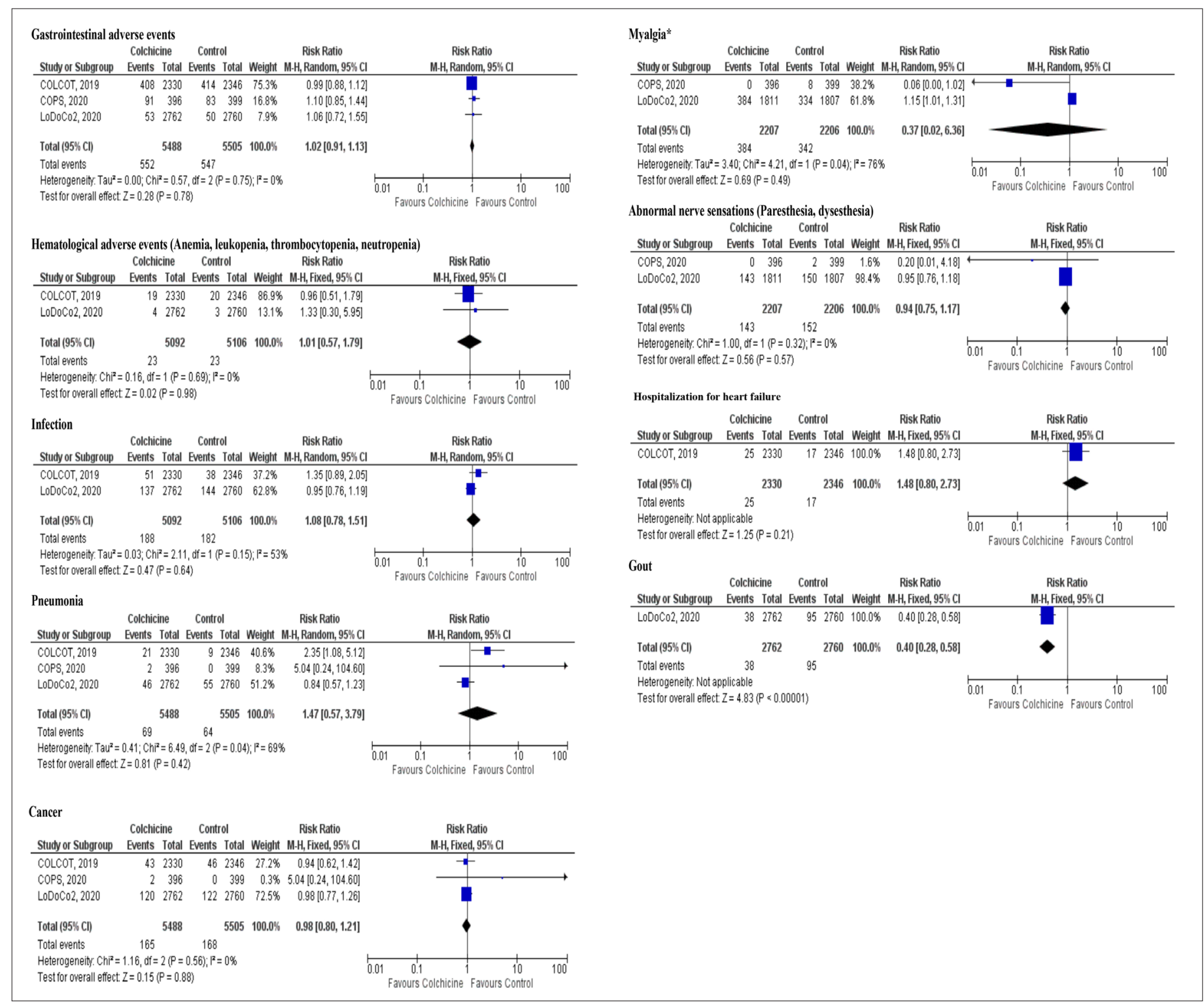

Figure 1. The incidences of adverse events in colchicine and control arms * In LoDoCo2 trial, myalgia was evaluated and reported in only Netherlands Cohort of the study. Meta-analysis results for the risk (pooled relative risks and $95 \% \mathrm{Cls}$ ) of adverse events. $\mathrm{Cl}$ - confidence interval

are major cornerstone drugs in atherosclerotic cardiovascular diseases and do not have an alternative in several conditions. Besides, the vast majority $(>94 \%)$ of the patients in the included studies were already on statin treatment. The safety analyses of these trials and the results of present meta-analysis did not alert about an increased risk of adverse events. Drug interaction between colchicine and statins are mainly based on an increased risk of muscle-related toxicity. From our point of view, real-world evidence is needed to reach a definitive conclusion as the reported data on muscle-related toxicity was very limited in all the clinical trials. For example, myalgia condition does not seem to be evaluated in the COLCOT trial (9) and was only evaluated in the Netherlands cohort of the LoDoCo2 trial (11). The given findings of the COPS study on myalgia was considerably different $(8$ events of myalgia in the placebo arm versus no event in the colchicine arm) (16); however, this study was conducted in a small sample size when compared to the COLCOT and LoDoCo2 trials. Moreover, there was no data on changes in creatine kinase (CK) activity in any of the studies. In daily practice, physicians mostly monitor patients who are using statins or other drugs with a potential risk of muscle-related toxicity risk with CK levels. Therefore, the changes in CK levels should be thoroughly investigated in future clinical trials. According to Medscape drug interaction checker, the interaction type between colchicine and all the statins were pharmacodynamic owing to the risk of muscle toxicity with both colchicine and statins. However, the pharmacokinetic mechanism through cytochrome (CYP) P450 can contribute to statin-related muscle toxicity as a result of possible 
Cardiovascular death

Colchicine Control Risk Ratio $\quad$ Risk Ratio

Study or Subgroup Events Total Events Total Weight M-H, Fixed, $95 \% \mathrm{Cl} \quad$ M-H, Fixed, $95 \% \mathrm{Cl}$

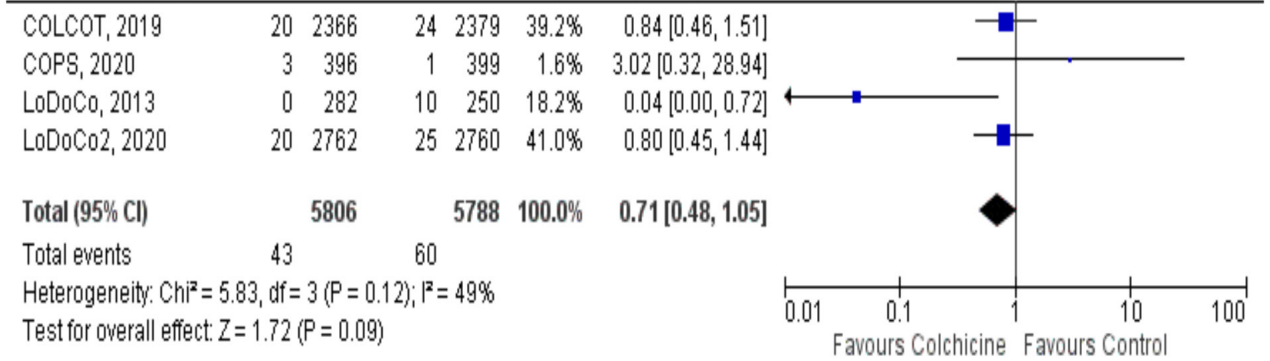

Non-cardiovascular death

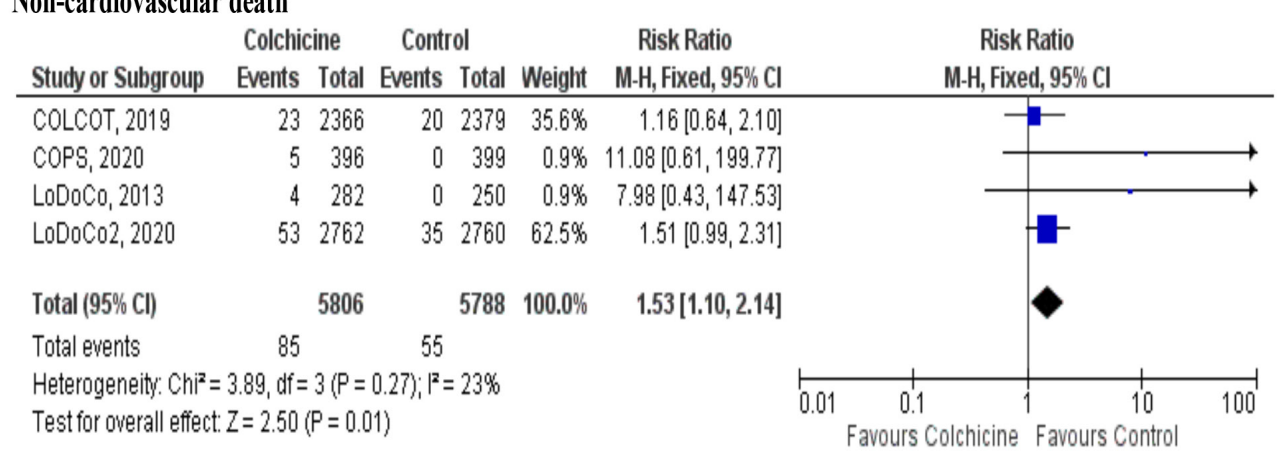

All-cause mortality

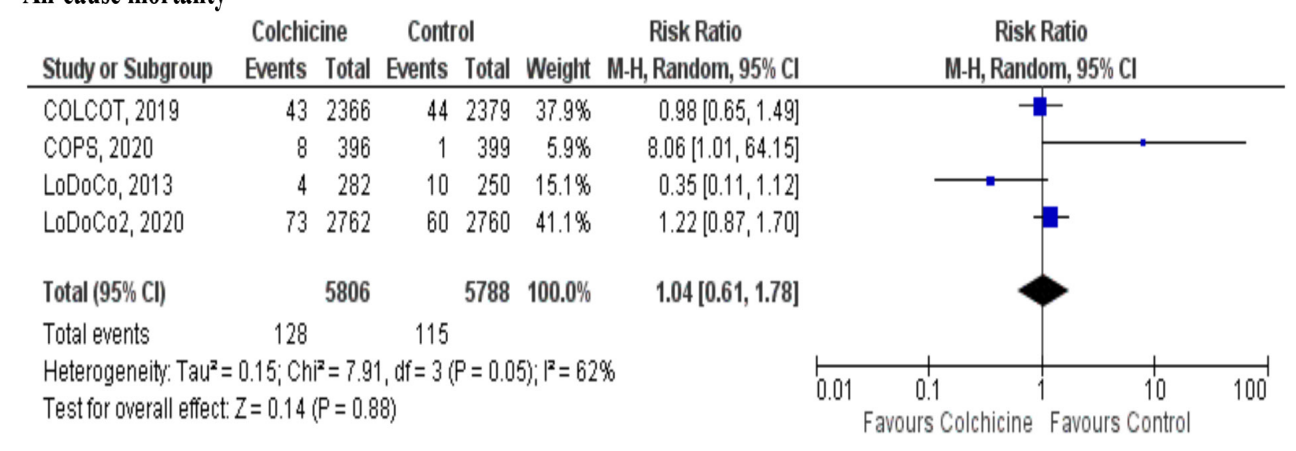

Figure 2. Comparison of the mortality outcomes

Meta-analysis results for the risk (pooled relative risks and 95\% Cls) of cardiovascular death, non-cardiovascular death and all-cause mortality. $\mathrm{Cl}$ - confidence interval

increase in the plasma level of statins. The metabolism of the statins is mainly based on CYP P450 system. Concomitant use of some drugs that interact with CYP P450 system, such as colchicine interaction with CYP3A4 can increase the risk of adverse events, including muscle-related toxicity. In this system, CYP3A4 is the major enzyme in the metabolism of simvastatin, atorvastatin, and lovastatin. Differently from all contemporary statins, fluvastatin metabolized by CYP2C9 and metabolism of pravastatin is very minimal by CYP3A4. Two other potent statins, rosuvastatin and pitavastatin, do not have a significant metabolism through the CYP P450 system (17). Until new data and evidence emerge, the use of low-dose colchicine $(0.5 \mathrm{mg} /$ day similar to the recent clinical trials), choosing of statins which do not have a significant drug interaction via CYP P450 system, and close monitoring of patients with both clinical findings and CK levels could be the basic recommendations.

Gastrointestinal symptoms, typically experienced as cramping, abdominal pain, and diarrhea are common side effects of colchicine treatment (18). However, the rate of gastrointestinal adverse events did not show a significant risk associated with colchicine treatment in this meta-analysis. Because of the heterogeneity of the included studies and low number of reported events, this finding may not exclude the risk of gastrointestinal adverse events. A previous meta-analysis that also included small size studies reported an increased risk of gastrointestinal adverse events, mainly diarrhea, with increasing doses (not with the low $0.5 \mathrm{mg} /$ day dose) with overall incidence $11 \%$ in patients receiving colchicine vs. $9.2 \%$ receiving placebo (19). Although 
low-dose colchicine $0.5 \mathrm{mg} /$ day does not seem to be excessively associated to an increased risk of gastrointestinal adverse events, this potential side effect should be considered during follow-up by physicians. In addition, in this meta-analysis, the rate of other evaluated serious complications including infection, pneumonia, cancer, hematological adverse events, abnormal nerve sensations and hospitalization for heart failure in the colchicine arm were not higher than control. However, further interpretation of these findings was not possible because of the low incidence of these adverse events and methodological differences in reporting across the included trials. Gout as an adverse event had only been evaluated in LoDoCo2 trial and regarding well-known use of colchicine in the treatment of gout, the gout incidence in the colchicine arm was significantly lower than control. This result may count as an additional benefit of colchicine treatment as the latest findings in this field has demonstrated that the high uric acid levels and gout can be a potential risk factor for cardiovascular disease, especially in female patients (20).

This meta-analysis mainly focused on the safety data from the included trials. Previous 3 meta-analyses pointed out that the use of colchicine can reduce the risk of major cardiovascular and cerebrovascular events in patients with coronary disease. In all these studies, colchicine was reported to be effective and safe in general $(19,21,22)$. In another meta-analysis published very recently, the authors concluded that low-dose colchicine might offer a significant reduction in major cardiovascular events, stroke, and need for revascularization in patients with acute coronary syndrome; whereas colchicine treatment might offer no significant reduction in the risk of ischemic events in patients with stable angina (23). In this metaanalysis, although the difference did not reach a significant level, the incidence of cardiovascular death tended to be lower compared with the control (RR: $0.71,95 \% \mathrm{Cl} 0.48-1.05 ; \mathrm{p}=0.09$ ). Similar to previous meta-analyses, we did not find a difference in the rate of all-cause mortality between the colchicine and control arms. One of the most remarkable findings in this metaanalysis was the significantly higher incidence of non-cardiovascular death in the colchicine arm compared with control (RR: $1.53,95 \% \mathrm{Cl} 1.10-2.14 ; \mathrm{p}=0.01$ ). To the best of our knowledge, our study is the first meta-analysis demonstrating a significantly higher incidence of non-cardiovascular death in the colchicine arm. The possible explanation of this finding could be the model we used. In all the previous meta-analyses $(19,21,22)$, a random-effects model was used for all the parameters evaluated. In contrast, both random-effects and fixed-effects models were used in our study owing to the considered heterogeneity of the medical condition. A fixed-effects model was used when heterogeneity was considered as low $\left(I^{2}<50 \%\right.$ and chi-squared $p$ value $>0.10$ ), as in cardiovascular death and non-cardiovascular death. Serious complications that may also be the cause of noncardiovascular death such as infection, cancer, pneumonia, and gastrointestinal events were similar between colchicine and control arms in this meta-analysis. Therefore, the reason of higher incidence of non-cardiovascular death should be investi- gated thoroughly as the existing data are not sufficient to reach a definitive conclusion.

\section{Study limitations}

Similar to the limitations of all meta-analyses, the aggregated data from published studies rather than the patient-level data was used. All the excluded studies were short-term and/or had small sample size. Therefore, we believe that their inclusion and low weight in the meta-analysis would not have a considerable influence on our results. Differing from other studies that were included, the LoDoCo trial (16) published in 2013, was not placebo-controlled and the rates of adverse events such as gastrointestinal adverse events, muscle related toxicity, and so forth, were not reported for the control arm; therefore, only the mortality outcomes of the LoDoCo study could be used in our work. In addition to the differences in reporting, the sample sizes were different across the included studies which affected their statistical weight and power in the meta-analysis. Furthermore, some of the reported adverse event incidences in the included studies showed significant heterogeneity as evidenced by high $\mathrm{I}^{2}$ and low chi-squared $p$ values. However, all those might be expected owing to the different protocols of the studies.

The adverse events were not evaluated as primary outcomes in all the included studies. The studies conducted on different patient populations (both patients with recent myocardial infarction and patients with chronic coronary disease) as well as the results were presented with the different definitions of the outcomes. The vast majority of the patients in all the included studies were older male patients. More research data is needed to focus on subgroup analysis in different patient populations.

\section{Conclusion}

Beyond its already approved indications, colchicine has potential to be effective in the treatment of atherosclerotic cardiovascular disease. However, cardiac patients using colchicine should be carefully monitored to avoid the complications of serious drug interactions. We believe that the findings presented in Tables 1 and 2 can provide a quick guide to estimate the risk. We would like to focus particularly on statins as it is very-well known that almost all the patients with coronary disease would need a statin as a cornerstone drug. From our point of view, the use of low-dose colchicine, choosing of statins which do not have a significant metabolism through CYP P450 system, and close monitorization of the patients can reduce the risk. According to our meta-analysis results, the use of low-dose colchicine was considered safe in general; and the findings were similar to previous meta-analyses, except for the noncardiovascular death incidence. Although it was not possible to make a further interpretation, the higher incidence of non-cardiovascular death in this meta-analysis cannot be ignored and requires a detailed investigation in further studies. Detailed subgroup analyses according to age, sex, medical condition, concomitant diseases, and drugs in future studies may also provide 
an opportunity to stratify and identify the patients who will benefit or be harmed from colchicine treatment.

Conflict of interest: None declared.

Peer-review: Externally peer-reviewed.

Author contributions: Concept - S.Ş., A.Y.Ü.; Design - S.Ş., E.K., C.B., Y.O.P., A.Y.Ü.; Supervision - S.Ş., A.Y.Ü.; Fundings - None; Materials S.Ş., E.K., C.B., Y.O.P., A.Y.Ü.; Data collection \&/or processing - S.Ş., E.K., C.B., Y.O.P.; Analysis \&/or interpretation - S.Ş., E.K., C.B., Y.O.P.; Literature search - S.Ş., E.K., C.B., Y.O.P.; Writing - S.Ş.; Critical review - S.Ş., E.K., C.B., Y.O.P., A.Y.Ü.

\section{References}

1. Hansson GK. Inflammation, atherosclerosis, and coronary artery disease. N Engl J Med 2005; 352: 1685-95. [Crossref]

2. Ridker PM, Everett BM, Pradhan A, MacFadyen JG, Solomon DH, Zaharris $E$, et al.; CIRT Investigators. Low-Dose Methotrexate for the Prevention of Atherosclerotic Events. N Engl J Med 2019; 380: 752-62. [Crossref]

3. Ridker PM, Everett BM, Thuren T, MacFadyen JG, Chang WH, Ballantyne C, et al.; CANTOS Trial Group. Antiinflammatory Therapy with Canakinumab for Atherosclerotic Disease. N Engl J Med 2017; 377: 1119-31. [Crossref]

4. Slobodnick A, Shah B, Krasnokutsky S, Pillinger MH. Update on colchicine, 2017. Rheumatology (Oxford) 2018; 57 (suppl_1): i4-11. [Crossref]

5. Andreu JM, Timasheff SN. Tubulin bound to colchicine forms polymers different from microtubules. Proc Natl Acad Sci USA 1982; 79: 6753-6. [Crossref]

6. Martínez GJ, Celermajer DS, Patel S. The NLRP3 inflammasome and the emerging role of colchicine to inhibit atherosclerosis-associated inflammation. Atherosclerosis 2018; 269: 262-71. [Crossref]

7. Fujisue K, Sugamura K, Kurokawa H, Matsubara J, Ishii M, Izumiya $\mathrm{Y}$, et al. Colchicine improves survival, left ventricular remodeling, and chronic cardiac function after acute myocardial infarction. Circ J 2017; 81: 1174-82. [Crossref]

8. Shah B, Allen N, Harchandani B, Pillinger M, Katz S, Sedlis SP, et al. Effect of Colchicine on Platelet-Platelet and Platelet-Leukocyte Interactions: a Pilot Study in Healthy Subjects. Inflammation 2016; 39: 182-9. [Crossref]

9. Tardif JC, Kouz S, Waters DD, Bertrand OF, Diaz R, Maggioni AP, et al. Efficacy and Safety of Low-Dose Colchicine after Myocardial Infarction. N Engl J Med 2019; 381: 2497-505. [Crossref]

10. Bouabdallaoui N, Tardif JC, Waters DD, Pinto FJ, Maggioni AP, Diaz $\mathrm{R}$, et al. Time-to-treatment initiation of colchicine and cardiovascular outcomes after myocardial infarction in the Colchicine Cardiovascular Outcomes Trial (COLCOT). Eur Heart J 2020; 41: 4092-9. [Crossref]
11. Nidorf SM, Fiolet ATL, Mosterd A, Eikelboom JW, Schut A, Opstal TSJ, et al.; LoDoCo2 Trial Investigators. Colchicine in Patients with Chronic Coronary Disease. N Engl J Med 2020; 383: 1838-47. [Crossref]

12. Drug interaction checker. Medscape [Last accessed: 30 May 2021] Available from: URL: https://reference.medscape.com/drug-interactionchecker.

13. Deeks JJ, Higgins JPT, Altman DG, (editors) on behalf of the Cochrane Statistical Methods Group. Chapter 9: Analysing data and undertaking meta-analyses. In: Higgins JPT, Green S (editors). Cochrane Handbook for Systematic Reviews of Interventions Version 5.1.0 (updated March 2011). The Cochrane Collaboration, 2011. Available from: URL: www.handbook.cochrane.org.

14. Moher D, Liberati A, Tetzlaff J, Altman DG; The PRISMA Group. Preferred reporting items for systematic reviews and meta-analyses: The PRISMA statement. PLoS Med 2009; 6: e1000097. [Crossref]

15. Tong DC, Quinn S, Nasis A, Hiew C, Roberts-Thomson P, Adams H, et al. Colchicine in Patients With Acute Coronary Syndrome: The Australian COPS Randomized Clinical Trial. Circulation 2020; 142: 1890-900. [Crossref]

16. Nidorf SM, Eikelboom JW, Budgeon CA, Thompson PL. Low-dose colchicine for secondary prevention of cardiovascular disease. $J$ Am Coll Cardiol 2013; 61: 404-10. [Crossref]

17. Schachter M. Chemical, pharmacokinetic and pharmacodynamic properties of statins: an update. Fundam Clin Pharmacol 2005; 19: 117-25. [Crossref]

18. Ben-Chetrit E, Levy M. Colchicine: 1998 update. Semin Arthritis Rheum 1998; 28(1): 48-59. [Crossref]

19. Andreis A, Imazio M, Piroli F, Avondo S, Casula M, Paneva E, et al. Efficacy and safety of colchicine for the prevention of major cardiovascular and cerebrovascular events in patients with coronary artery disease: a systematic review and meta-analysis on 12869 patients. Eur J Prev Cardiol 2021: zwab045. [Crossref]

20. Muiesan ML, Agabiti-Rosei C, Paini A, Salvetti M. Uric Acid and Cardiovascular Disease: An Update. Eur Cardiol 2016; 11: 54-9. [Crossref]

21. Fiolet ATL, Opstal TSJ, Mosterd A, Eikelboom JW, Jolly SS, Keech $A C$, et al. Efficacy and safety of low-dose colchicine in patients with coronary disease: a systematic review and meta-analysis of randomized trials. Eur Heart J 2021; 42: 2765-75. [Crossref]

22. Xia M, Yang X, Qian C. Meta-analysis Evaluating the Utility of Colchicine in Secondary Prevention of Coronary Artery Disease. Am J Cardiol 2021; 140: 33-8. [Crossref]

23. Ullah W, Haq S, Zahid S, Gowda SN, Ottman P, Saleem S, et al. Safety and Efficacy of Colchicine in Patients with Stable CAD and ACS: A Systematic Review and Meta-analysis. Am J Cardiovasc Drugs 2021 Jun 21. doi: 10.1007/s40256-021-00485-7. [Epub ahead of print] [Crossref] 


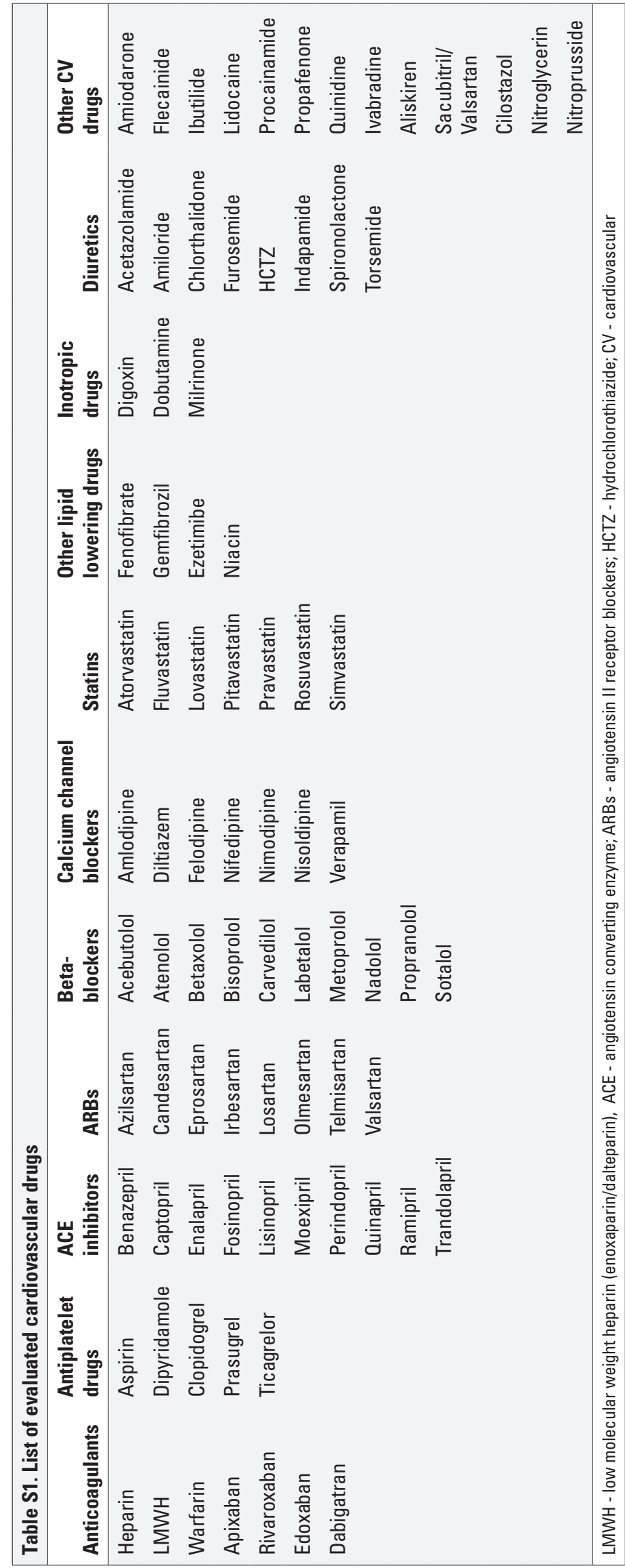

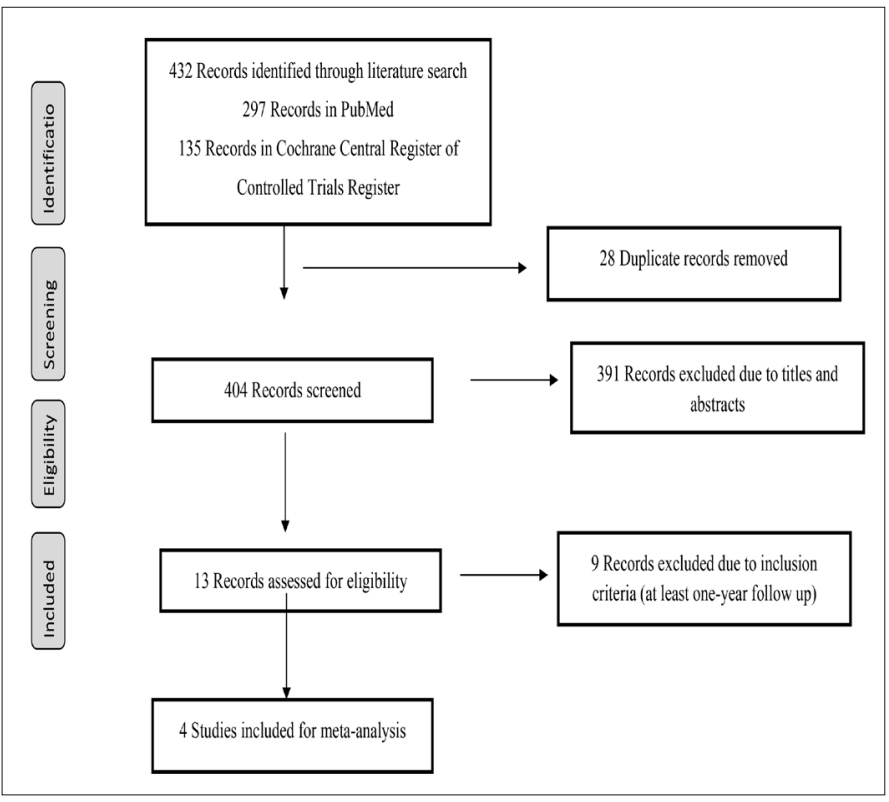

Figure S1. Study flow chart

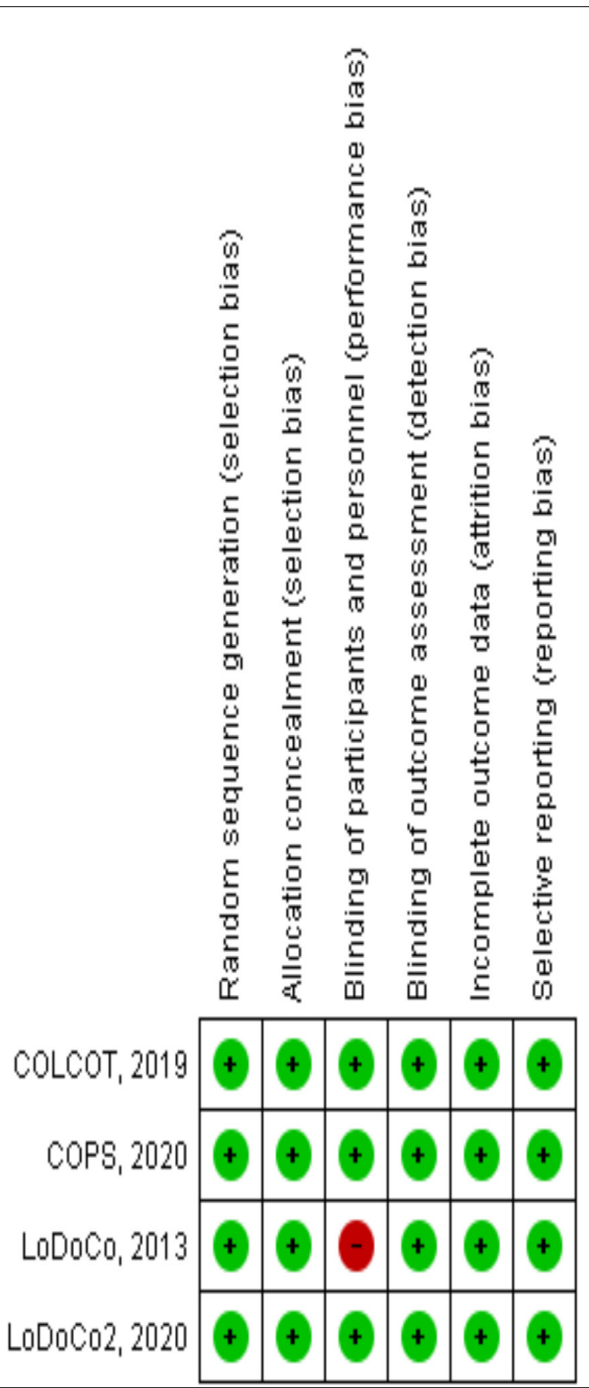

Figure S2. The risk of bias assessment 